Classification

Physics Abstracts

$61.30 \mathrm{~B}-64.60 \mathrm{C}-64.70 \mathrm{M}$

\title{
Demixing in a hard rod-plate mixture
}

\author{
René van Roij and Bela Mulder \\ FOM-Institute for Atomic and Molecular Physics (AMOLF), Kruislaan 407, \\ 1098 SJ Amsterdam, The Netherlands
}

(Received 5 April 1994, accepted 1 July 1994)

\begin{abstract}
We argue that the possibility to observe a stable biaxial nematic phase in a binary mixture of prolate and oblate hard particles is seriously limited by the existence of entropydriven demixing. This result follows from a simple Onsager-type density functional theory. An important feature is the coupling of the demixing mechanism to the orientational order of the system. The strength of this coupling is dependent on the asphericity of the particles, and is directly related to the stability of the biaxial nematic phase.
\end{abstract}

\section{Introduction.}

Mixtures of rod- and plate-like particles are interesting because they may exhibit a biaxial nematic phase that is absent in the pure components. In this liquid crystalline phase both species are orientationally ordered, but in mutually perpendicular directions. The possible existence of the biaxial nematic phase may explain why this system attracted a lot of attention in the past [1-7]. In the only study devoted to lyotropic (hard core) rod-plate mixtures that took into account the full set of thermodynamic equilibrium conditions, the biaxial phase was found to be thermodynamically stable for the particle shapes considered [7]. This was consistent with the accepted belief that hard particles are miscible in any proportion.

Recent theoretical developments in the study of binary hard sphere mixtures, however, seem to indicate that phase separation does occcur in this system if the diameter ratio of the spheres is sufficiently large $[8,9]$. Moreover, very recent computer simulations clearly show a demixing transition in several hard core mixtures [10]. The mechanism of these demixing transitions is the so-called depletion effect: the gain of configurational entropy of small particles due to excluded volume overlap of clustered large particles outweighs the loss of configurational entropy of these large particles. Note that depletion is essentially a three-or-more body effect.

Inspired by these new developments we have reconsidered the stability of the hard rod-plate mixture. Surprisingly, all ingredients for a demixing transition turn out to be present even if the volume of the rods equal that of the plates, which rules out the depletion mechanism. The driving force in this case is the excess average excluded volume of a rod-plate pair as compared 
to that of a rod-rod or plate-plate pair. Consequently we are dealing with a two-body effect, which can be treated in the hard particle equivalent of mean-field theory: the Onsager- or second virial approximation. An important feature of the theory is the coupling of the demixing mechanism to the orientational ordering of the system. This coupling is determined by the aspect ratio of the rods and the plates, which is the only relevant microscopic parameter. In turn, this aspect ratio determines the density-range in which the biaxial phase is stable.

In the following we first describe hard core rod-plate mixtures in terms of a free energy expression. Then we discuss the excluded volume interactions qualitatively, on the basis of which we can already predict a demixing transition. We proceed with a quantitative numerical study of an explicit model, which confirms the qualitative predictions.

\section{Free energy functional.}

We consider a hard particle mixture of $N_{\mathrm{r}}$ rods and $N_{\mathrm{p}}$ plates in a three-dimensional volume $V$. We define the total number of particles $N=N_{\mathrm{r}}+N_{\mathrm{p}}$, the total density $\rho=N / V$ and the mole fraction of rods and plates $x_{\mathrm{r}}=N_{\mathrm{r}} / N$ and $x_{\mathrm{p}}=N_{\mathrm{p}} / N$, respectively.

We characterize the particles involved by four parameters: the length $\ell$ and diameter $d$ of the rods (with $\ell>d$ ) and the width $w$ and the height $h$ of the plates (with $w>h$ ). The generic shape of the particles is depicted in figure 1. It will turn out that other details of the exact shape of the particles are irrelevant for the general argument, provided the particles are sufficiently aspherical, i.e. their aspect ratios $\ell / d$ and $w / h$ large enough.

A specific system is described by a free energy functional $\mathcal{F}$ of the single particle orientation distribution functions $\psi_{\mathrm{r}}$ and $\psi_{\mathrm{p}}$ of the rods and the plates, respectively. The thermodynamic equilibrium value of the free energy is the minimum value of $\mathcal{F}$ for given composition and density. The equilibrium orientation distribution functions are those which minimize $\mathcal{F}$. We write $\mathcal{F}$ as a virial series which we truncate after the second virial coefficient: the so-called Onsager approximation [11]. This approximation, although in principle only exact in the isotropic phase of a system of extremely long rods, can be interpreted as the equivalent of mean-field for hard particle systems and is expected to give qualitatively or semi-quantitatively correct results $[12,13]$. The free energy expression consists of the ideal gas contribution, the entropy of mixing, the orientational entropy and an excess part due to the excluded volume

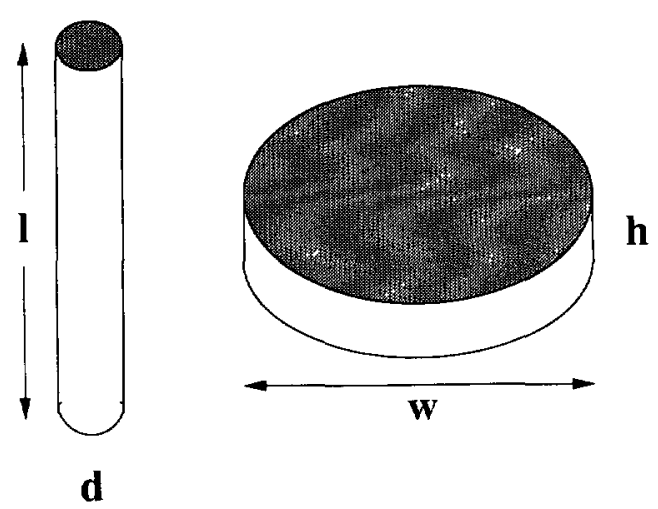

Fig. 1. - Dimensions characterizing generic rod- and plate-like particles. 
interactions:

$$
\begin{aligned}
\frac{\beta \mathcal{F}}{N} & =\log \left(\rho \mathcal{V}_{T}\right)-1 \\
& +x_{\mathrm{r}} \log x_{\mathrm{r}}+x_{\mathrm{p}} \log x_{\mathrm{p}} \\
& +x_{\mathrm{r}} \int \mathrm{d} \Omega \psi_{\mathrm{r}}(\Omega) \log \psi_{\mathrm{r}}(\Omega)+x_{\mathrm{p}} \int \mathrm{d} \Omega \psi_{\mathrm{p}}(\Omega) \log \psi_{\mathrm{p}}(\Omega) \\
& +\frac{\rho}{2}\left(x_{\mathrm{r}}^{2}\left\langle E^{(\mathrm{rr})}\right\rangle+2 x_{\mathrm{r}} x_{\mathrm{p}}\left\langle E^{(\mathrm{rp})}\right\rangle+x_{\mathrm{p}}^{2}\left\langle E^{(\mathrm{pp})}\right\rangle\right)
\end{aligned}
$$

where we used the shorthand notation

$$
\left\langle E^{\left(\sigma \sigma^{\prime}\right)}\right\rangle=\int \mathrm{d} \Omega \int \mathrm{d} \Omega^{\prime} \psi_{\sigma}(\Omega) E^{\left(\sigma \sigma^{\prime}\right)}\left(\Omega, \Omega^{\prime}\right) \psi_{\sigma^{\prime}}\left(\Omega^{\prime}\right)
$$

Here $\beta$ is the inverse temperature in units of $\left(k_{\mathrm{B}}\right)^{-1}$ and $\mathcal{V}_{\mathrm{T}}$ is the product of the relevant thermal wavelengths. The particle orientation is denoted by $\Omega$ and the orientation dependent excluded volume of a $\sigma \sigma^{\prime}$ pair by $E^{\left(\sigma \sigma^{\prime}\right)}\left(\Omega, \Omega^{\prime}\right)$, where $\sigma$ denotes the species and takes the "values" $\mathrm{r}$ (rod) and $\mathrm{p}$ (plate).

The phase transitions in the mixture are determined by competitions between the non-ideal gas terms of $\mathcal{F}$. The entropy of mixing favors a mixed phase and the orientational entropy an orientationally disordered (isotropic) phase. If any transition takes place in the system, it must be due to the excluded volume interaction terms in (1) competing with either the entropy of mixing or the orientational entropy or both. Since the excluded volume terms are proportional to $\rho$, their importance grows when the density is increased. We will now formulate criteria for the density above which the excluded volume terms are dominant over (one of the) entropy terms.

The key ingredient for the determination of $\rho_{\text {or }}$, the density where the system changes from mixed and disordered to mixed and orientationally ordered, is the observation that the orientational entropy in the isotropic phase is of order unity (in units of $k_{\mathrm{B}}$ ). Hence for densities larger than

$$
\rho_{\text {or }} \sim\left(\max _{\sigma \sigma^{\prime}}\left\{x_{\sigma} x_{\sigma^{\prime}}\left\langle E^{\left(\sigma \sigma^{\prime}\right)}\right\rangle_{\text {iso }}\right\}\right)^{-1}
$$

the gain in translational entropy due to the decreasing excluded volume will offset the loss of orientational entropy. Here $\langle. .\rangle_{\text {iso }}$ stands for an orientational average in the isotropic phase, see (2).

In order to estimate $\rho_{\mathrm{ps}}$, the density where the system tends to phase separate, we consider the free energy difference $\Delta \mathcal{F}$ of a mixed phase and pure rod and plate phases with weights $x_{\mathrm{r}}$ and $x_{\mathrm{p}}$, where we assume equal orientational order of the species in all the phases:

$$
\frac{\beta \Delta \mathcal{F}}{N}=x_{\mathrm{r}} \log x_{\mathrm{r}}+x_{\mathrm{p}} \log x_{\mathrm{p}}+\chi x_{\mathrm{r}} x_{\mathrm{p}}
$$

The interaction parameter $\chi$ is defined as

$$
\chi=\frac{\rho}{2}\left(2\left\langle E^{(\mathrm{rp})}\right\rangle-\left\langle E^{(\mathrm{rr})}\right\rangle-\left\langle E^{(\mathrm{pp})}\right\rangle\right)
$$

Straightforward analysis of (4) shows a tendency to demix when $\chi$ exceeds a critical value $\chi_{\mathrm{ps}}=1 /\left(2 x_{\mathrm{r}} x_{\mathrm{p}}\right)$. In that case the orientation averaged excluded volume of a rod-plate pair is sufficiently more unfavorable than that of rod-rod and plate-plate pairs. Thus for the mixtures of interest, which consist of a comparable number of rods and plates, we have

$$
\rho_{\mathrm{ps}} \sim\left(2\left\langle E^{(\mathrm{rp})}\right\rangle-\left\langle E^{(\mathrm{rr})}\right\rangle-\left\langle E^{(\mathrm{pp})}\right\rangle\right)^{-1}
$$


Note that the orientational order is not specified in expression (6); it holds for both the isotropic and the orientationally ordered phases.

\section{Excluded volume.}

Since we are interested in particles of different shape rather than size, we set the volume of the rods and the plates equal. In terms of the microscopic lengths as introduced before this means that

$$
\ell d^{2}=h w^{2} \approx v
$$

where the particle volume $v$ is defined upto a numerical factor of order unity, which is different for different particle geometries. This leaves the aspect ratios of the particles as the only relevant microscopic parameters. For simplicity we consider only the symmetric case, with the common aspect ratio $\kappa>1$ the only free parameter left:

$$
\frac{\ell}{d}=\frac{w}{h} \equiv \kappa
$$

and hence

$$
\begin{aligned}
\ell & =h \kappa^{4 / 3} \\
w & =h \kappa \\
d & =h \kappa^{1 / 3} \\
v & \approx h^{3} \kappa^{2}
\end{aligned}
$$

As both the ordering and the demixing are governed by the orientation averaged excluded volumes, we need to calculate these and we will do so in two extreme cases of interest: (i) both species orientationally disordered (isotropic phase) and (ii) both species totally ordered in mutually perpendicular directions (biaxial phase). We make use of Onsager's expression for the excluded volume of two arbitrary cylinders with fixed relative orientations [11]. For $\kappa \gg 1$ the dominant terms in the expression for the excluded volume of two uniaxial particles will be the same upto numerical factors of order unity. For $\kappa \gg 1$ we find:

$$
\begin{array}{lll}
\left\langle E^{(\mathrm{rr})}\right\rangle_{\text {so }} \sim h^{3} \kappa^{3} & \left\langle E^{(\mathrm{rr})}\right\rangle_{\text {or }} \sim h^{3} \kappa^{2} \\
\left\langle E^{(\mathrm{pp})}\right\rangle_{\text {Iso }} \sim h^{3} \kappa^{3} & \left\langle E^{(\mathrm{pp})}\right\rangle_{\text {or }} \sim h^{3} \kappa^{2} \\
\left\langle E^{(\mathrm{rp})}\right\rangle_{\text {iso }} \sim h^{3} \kappa^{10 / 3} & \left\langle E^{(\mathrm{rp})}\right\rangle_{\text {or }} \sim h^{3} \kappa^{8 / 3}
\end{array}
$$

where $\langle\ldots\rangle_{\text {iso,or }}$ stands for an orientation average in the isotropic and the completely orientationally ordered phase, respectively, see (2). One immediately sees that the excluded volume of a rod-plate pair, both in the isotropic and in the completely ordered phase, is larger than that of a rod-rod or a plate-plate pair in the same phase. From the ordering and demixing criteria (3) and (6) we see that it is the rod-plate excluded volume interaction which is responsible for both the ordering and the demixing. Introducing the packing fraction $\eta=\rho v$ and inserting the results of (10) into (3) and (6) we obtain for $\kappa \gg 1$ :

$$
\eta_{\mathrm{or}}=\rho_{\mathrm{or}} v \sim \kappa^{-4 / 3}
$$

and

$$
\eta_{\mathrm{ps}}=\rho_{\mathrm{ps}} v \sim \begin{cases}\kappa^{-4 / 3} & \text { in isotropic phase } \\ \kappa^{-2 / 3} & \text { in ordered phase }\end{cases}
$$

The most striking observation is that the system does demix at sufficiently high packing fractions, although whether this demixing takes place in the isotropic phase or in the ordered phase is undetermined, as is the coupling to the orientational ordering transition. If the system orders before it demixes, we can conclude that the ordered state is stable in a finite range of packing fractions, since then $\eta_{\mathrm{ps}} / \eta_{\mathrm{or}} \sim \kappa^{2 / 3} \gg 1$. 


\section{Explicit model.}

Our general argument is based on the Helmholtz free energy difference of mixed and completely demixed phases at the same density. However, coexisting phases need neither be pure nor equally dense. The correct procedure is to solve the full set of thermodynamic equilibrium conditions, implying in this case equal pressure and equal chemical potentials in the coexisting phases. Another reason for a full calculation is to elucidate how the ordering and demixing transitions are coupled.

A full calculation requires a specific model system. For simplicity we represent the rods and plates by rectangular blocks of size $\ell \times d \times d$ and $h \times w \times w$. We discretize the orientations of the particles such that both the major and the minor axes can only point into the three mutually perpendicular directions of the lab frame. Hence we distinguish three possible particle orientations. This model can therefore be considered a direct extension of the $\mathrm{Zwanzig}$ rod model [14]. Because of the discretized orientations and the four-fold symmetry of the particles around their main axis, there are only two different relative orientations for a pair of particles: parallel and perpendicular. Hence for every $\sigma \sigma^{\prime}$ pair there are only two possible excluded

volumes: $E_{\|}^{\left(\sigma \sigma^{\prime}\right)}$ and $E_{\perp}^{\left(\sigma \sigma^{\prime}\right)}$ for parallel and perpendicular orientations, respectively. Again we consider rods and plates of equal volume and aspect ratio, so that the lengths are related as in (9). It is an easy exercise to check that also in this case the scaling relations of the excluded volumes for large $\kappa$ are given in expression (10). We therefore expect orientational ordering and demixing in this system as predicted by the general theory.

We solved numerically the full set of thermodynamic equilibrium conditions of this system in the Onsager approximation for several values of $\kappa$. We find four possible phases. At low densities we find the isotropic I phase, at higher densities we distinguish three nematic phases: the uniaxial rod-like nematic $N^{(+)}$, the uniaxial plate-like nematic $N^{(-)}$and the biaxial nematic B phase.

We depict the phase diagram in the $\eta-x_{\mathrm{r}}$ plane for $\kappa=5$ in figure $2 \mathrm{a}$. The phase boundaries are denoted by thick curves. The thin straight lines denote coexistence between the end points. We see for $x_{\mathrm{r}} \neq 1 / 2$ a first order isotropic-uniaxial nematic transition, which softens down to a continuous transition at $x_{\mathrm{r}}=1 / 2$. Although there is a biaxial phase between the two uniaxially ordered phases, it is unstable with respect to phase separation into coexisting $N^{(-)}$and $N^{(+)}$ phases. For this value of $\kappa$ we find clearly that $\eta_{\mathrm{or}}=\eta_{\mathrm{ps}}$, so that the demixing and the ordering transition are strongly coupled.

We also performed the analogous calculations for $\kappa=15$, which yield the phase diagram as depicted in figure $2 \mathrm{~b}$. Again the low density phase is isotropic, while we find three stable orientationally ordered phases at higher densities. At sufficiently high densities, however, the $B$ phase is unstable with respect to uniaxial-uniaxial phase separation. The uniaxial-biaxial transitions are continuous (when $B$ is stable). For $\kappa=15$ we clearly have $\eta_{\text {or }}<\eta_{\mathrm{ps}}$, so that the orientational ordering and the demixing are decoupled.

The critical aspect ratio $\kappa_{\mathrm{c}}$ separating the two regimes is estimated to be $\kappa_{\mathrm{c}}=8.80 \pm 0.01$ in this case. We expect a similar cross-over aspect ratio in other systems, although the exact value will depend on the details of the model.

\section{Conclusions and discussion.}

In this paper we have given both qualitative and quantitative arguments for demixing in binary mixtures of hard rods and plates of equal volume and aspect ratio. From the qualitative analysis it is predicted that the system demixes into a rod-rich and a plate-rich phase at high densities, if the particles are sufficiently aspherical. On the basis of the qualitative arguments we can 

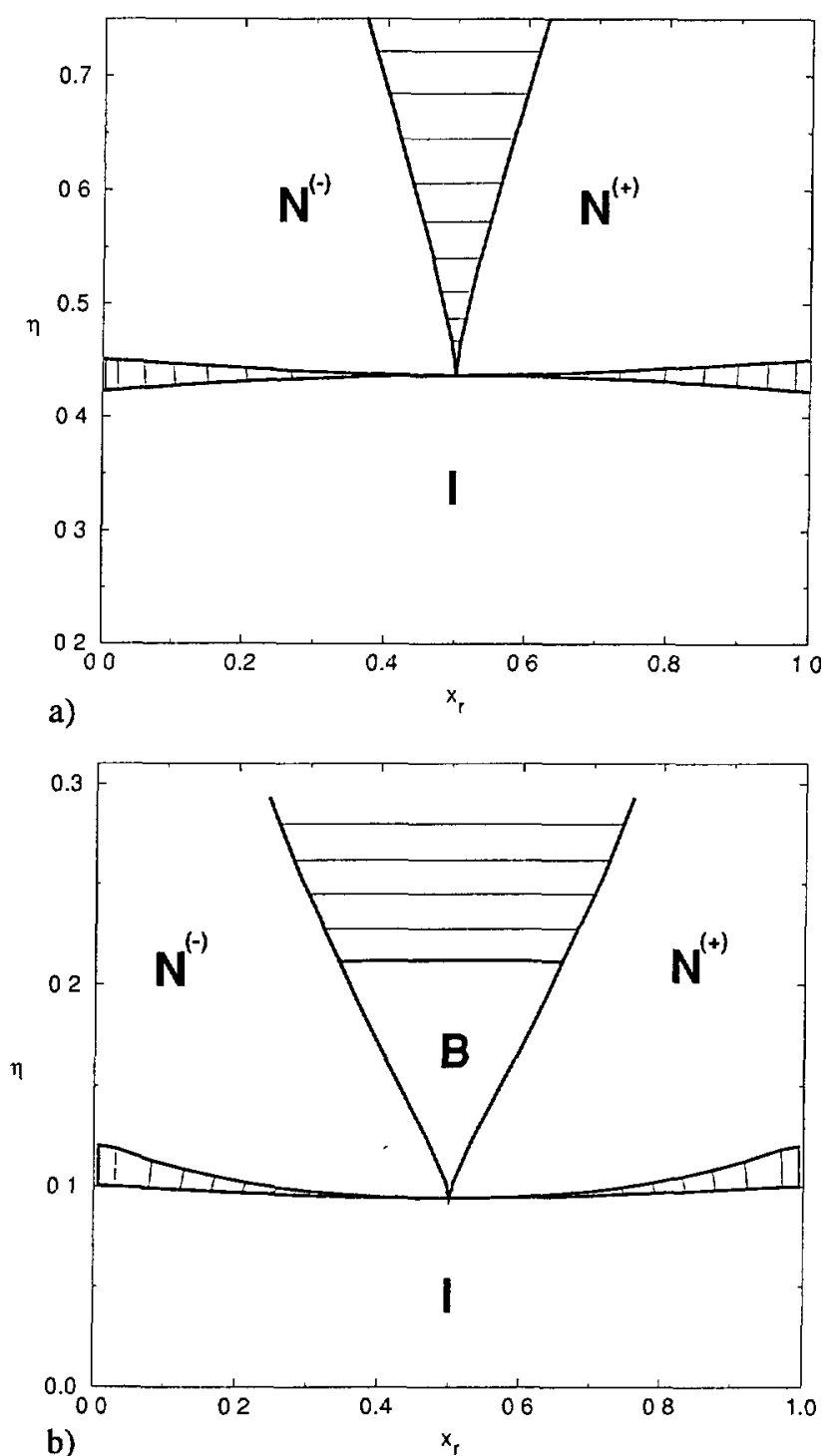

Fig. 2. - Phase diagram in $\eta-x_{\mathrm{r}}$ plane of a system of rectangular blocks with discretized orientations. Here $\eta$ is the packing fraction and $x_{\mathrm{r}}$ the mole fraction of rods. The thick curves represent the phase boundaries, the thin lines denote phase coexistence between the endpoints. I: isotropic, $N^{(+)}$. uniaxial rod-like nematic, $N^{(-)}$. uniaxial plate-like nematic and B: biaxial nematic. (a) $\kappa=5$. (b) $\kappa=15$.

not determine whether the demixing takes place in the orientationally ordered or disordered phase. A quantitative analysis of a simple model system reveals that the ordering and demixing transition occur at the same density if the aspect ratio of the particles is smaller than a critical one. For sufficiently aspherical particles the system orders at a density which is smaller than the demixing density, leaving a finite density range for a stable biaxial nematic phase.

In fact the theory presented here is very similar to the classical mean-field treatment of a dense binary mixture on a lattice [15], where the roles of temperature and interaction energies 
are now played by inverse density and excluded volumes, respectively. In this view it should not come as a surprise that our results are consistent with those of mean-field descriptions of thermotropic rod-plate mixtures, which also show a possible instability of the biaxial nematic phase [4-6].

Let us now discuss the approximations of the theory and their possible influence on the results. The first essential approximation we made is the truncation of the free energy functional after the second virial coefficient. We have, however, also performed calculations on the discretized orientation model in the third virial approximation. All major results of the Onsager approximation are found to be robust against the inclusion of this higher order correction, showing that the behavior is not an artefact of the pair approximation. The second essential approximation is the restriction to spatially homogeneous phases in the free energy functional. To our knowledge it is an open question what kind of inhomogeneous phases rod-plate mixtures form at high densities. We do certainly expect smectic- or solid-like order at packing fractions exceeding $\eta \approx 0.5$, analogous to the fluid-solid and the nematic-smectic transition in the case of pure hard spheres and spherocylinders, respectively. For $\kappa \geq 15$ the phenomena described here take place well below this packing fraction. For $\kappa=5$ the inhomeogeneous phases may interfere with the phase behavior we found, so that more research is required to map out the actual phase diagram.

\section{Acknowledgments.}

The work of the FOM Institute is part of the research program of FOM and is made possible by financial support from the Netherlands Organization for Scientific Research (NWO). It is a pleasure to thank Henk Lekkerkerker for valuable and helpful comments and Daan Frenkel for a careful reading of the manuscript.

\section{References}

[1] Alben R., J. Chem. Phys. 59 (1973) 4299.

[2] Rabin Y., McMullen W.E. and Gelbart W.M., Mol. Cryst. Liq. Cryst. 89 (1982) 67.

[3] Chen Z.Y. and Deutch J.M., J. Chem. Phys. 80 (1984) 2151.

[4] Hashim R., Luckhurst G.R. and Romano S., Mol. Phys. 53 (1984) 1535.

[5] Palffy-Muhoray P., de Bruyn J.R. and Dunmur D.A., J. Chem. Phys. 82 (1985) 5294.

[6] Sharma S.R., Palffy-Muhoray P., Bergersen B. and Dunmur D.A., Phys. Rev. A 32 (1985) 3752.

[7] Stroobants A. and Lekkerkerker H.N.W., J. Phys. Chem. 88 (1984) 3669.

[8] Biben T. and Hansen J.P., Phys. Rev. Lett. 66 (1991) 2215.

[9] Lekkerkerker H.N.W. and Stroobants A., Physica A 195 (1993) 387.

[10] Dijkstra M. and Frenkel D., Phys. Rev. Lett. 72 (1994) 298.

[11] Onsager L., Ann. N.Y. Acad. Sci. 51 (1949) 627.

[12] Frisch H.L., Rivier N. and Wyler D., Phys. Rev. Lett. 54 (1985) 2061.

[13] Kirkpatrick T.R., J. Chem. Phys. 85 (1986) 3515.

[14] Zwanzig R., J. Chem. Phys. 39 (1963) 1714.

[15] See for instance: Hill T.L., An introduction to statistical thermodynamics (Dover Publications, New York) pp 246 and pp 371. 